\title{
ATIVIDADE DE INFERÊNCIA INFORMAL PARA AVALIAR $\pi$
}

\section{INFORMAL INFERENCE ACTIVITY TO ESTIMATE $\pi$}

\author{
Marcos Nascimento Magalhães ${ }^{1}$
}

\begin{abstract}
Resumo: Inferência é a parte da Estatística que trata de previsões, para uma população, a partir das informações de uma amostra. $\mathrm{O}$ assunto é discutido de maneira formal em disciplinas de Estatística de cursos superiores, pois envolve conceitos matemáticos avançados. Entretanto, fazer previsões é algo presente na vida de todos e com os estudantes da Educação Básica não é diferente. É importante que os estudantes desse nível de ensino tenham oportunidade de fazer previsões, ainda que limitadas e informais, como uma experiência inicial do uso dessa parte fundamental da Estatística. Neste artigo, aproveitamos a mística envolvendo o número $\pi$ para discutir, de modo informal, aspectos da Inferência Estatística. Descrevemos uma atividade, presente no portal AtivEstat-Atividades de Estatística, que usa conceitos simples de Geometria para avaliar o valor do número irracional $\pi$.
\end{abstract}

Palavras-chave: Inferência informal; Atividade; Valor de $\pi$.

\begin{abstract}
Inference is the part of Statistics that deals with forecasts for a population from the information in a sample. The subject is discussed formally in higher education statistics disciplines as it involves advanced mathematical concepts. However, making predictions is something present in everyone's life and with students at theBasic Education it is not different. It is important that students, at this educational level, have the opportunity to make predictions, even if they are limited and informal, as an initial experience of using this key part of Statistics. In this article, we take advantage of the mystique involving the number $\pi$, to informally discuss aspects of Statistical Inference. We describe an activity, included in the Portal AtivEstat - Statistics Activities, that uses simple concepts of Geometry to evaluate the value of the irrational number $\pi$.
\end{abstract}

Keywords: Informal inference; Activity; $\pi$ value.

\section{Introdução}

O objetivo deste artigo, com característica de ensaio ou estudo, é ressaltar a importância de discutir a Inferência Estatística Informal na Educação Básica e apresentar uma atividade para obter estimativas de $\pi$, fazendo sua conexão com a teoria envolvida.

O número $\pi$ tem fascinado estudantes e pesquisadores e tem uma longa história no desenvolvimento humano (BOYLE, 1996). Para seu estabelecimento como constante matemática foi necessário o reconhecimento de que existe, em qualquer círculo, uma razão constante entre comprimento e diâmetro. Segundo a Wikipédia ${ }^{2}$, Arquimedes de Siracusa (287-212 AC) foi um dos primeiros a calcular limites para seu valor usando o

${ }^{1}$ Doutor Industrial Engeneering And Operation Research pela Virginia Polytechnic Institute and State University (VaTech). Professor associado do Departamento de Estatística do Instituto de Matemática e Estatística da USP, São Paulo, SP, Brasil, Email: marcos@ime.usp.br

${ }^{2}$ Disponível em https://pt.wikipedia.org/wiki/Pi. Acesso em: 10 maio. 2019. 
DOI: http://dx.doi.org/10.33238/ReBECEM.2019.v.3.n.2.22509

perímetro de polígonos regulares inscritos no círculo (para o limite inferior) e circunscritos ao círculo (para o limite superior). A busca por melhores aproximações acompanhou a evolução da ciência e hoje, devido ao computador, a precisão pode chegar ao trilhão de casas decimais. Ainda com base na referência indicada, a primeira conexão entre Estatística e o valor de $\pi$ foi feita por Georges de Buffon no século XVIII, com um método conhecido como Agulha de Buffon, que voltaremos a comentar mais adiante.

As recomendações oficiais, como os Parâmetros Curriculares Nacionais (BRASIL, 1997, 1998, 2000, 2002) e a Base Nacional Comum Curricular- BNCC (BRASIL, 2017), apresentam indicações de tópicos e formas de abordagem da Matemática nas várias séries da Educação Básica. A conexão entre diferentes assuntos é recomendada nesses textos como indica, por exemplo, a BNCC:

\begin{abstract}
A Base Nacional Comum Curricular, no tocante à Matemática, também se aproxima dos Parâmetros Curriculares Nacionais, tendo em vista que esses documentos visam à construção de um referencial que oriente a prática escolar de forma a contribuir para que todos os estudantes brasileiros tenham acesso a um conhecimento matemático que lhes possibilite, de fato, sua inserção, como cidadãos, no mundo do trabalho, das relações sociais e da cultura.

Para essa inserção, o ensino de Matemática deve contribuir para que os estudantes façam observações sistemáticas de aspectos quantitativos e qualitativos da realidade, estabelecendo inter-relações entre eles, utilizando conhecimentos relativos à aritmética, à geometria, às medidas, à álgebra, à estatística e à probabilidade. Desse modo a Matemática poderá fornecer ferramentas para a compreensão da realidade e nela atuar, e desenvolver formas de raciocínio e processos (BRASIL, 2017, p. 134).
\end{abstract}

A Estatística tem uma vocação quase que natural para se conectar a diferentes assuntos. Na Educação Básica brasileira, a Estatística é parte da disciplina de Matemática e pode possibilitar interações produtivas com disciplinas e mesmo com outros tópicos de Matemática. Em anos recentes, ampliou sua presença devido ao crescimento acentuado do número de consumidores de informações estatísticas. Argumentos na imprensa, e em diversas outras áreas, sempre incluem algum dado que dá suporte às conclusões apresentadas. No Brasil, outra contribuição importante é dada pela comunidade acadêmica brasileira da área de Educação Estatística que têm aumentado sua visibilidade com publicações e participações em congressos, bem como influenciado a legislação educacional do país.

Em Estatística a parte de Inferência é central como indica Garfield e Zvi (2008) e sua apresentação e discussão e feita em disciplinas de cursos superiores. Na última década, pesquisadores têm sugerido abordar, previamente, mesmo na Educação Básica, as ideias inferenciais por meio do que ficou denominado Inferência Estatística Informal. Trata-se, como descreveremos melhor adiante, da utilização de mecanismos informais 




DOI: http://dx.doi.org/10.33238/ReBECEM.2019.v.3.n.2.22509
Revista Brasileira de Educação em

Ciências e Educação Matemática

para construir inferências. Nesses contextos, não se usa o rigor matemático tradicional da Inferência e explora-se a intuição para obter estimativas.

Em nossa vida cotidiana, estamos sempre envolvidos em fazer estimativas, desde as mais simples e informais, como o tempo dos trajetos diários entre casa e trabalho, até algumas mais importantes como o tempo de recuperação de uma doença ou os gastos em viagem de férias da família. Também, nas manhãs das grandes cidades, a previsão do tempo auxilia na escolha da roupa e no planejamento do dia. Várias dessas previsões são resultados de modelos estatísticos e aparecem, para o grande público, como afirmações categóricas.

Apesar de muitas das previsões fazerem uso de técnicas refinadas da Inferência Formal, o apelo por uma maior comunicação reduz detalhes importantes que, às vezes, propiciam interpretações equivocadas. Por exemplo, se a previsão aponta uma probabilidade de chuva de $90 \%$, ao não chover, alguns podem considerar que a previsão foi errada. Nessa direção, a Matemática da escola pode se aproximar do dia a dia da sociedade para problematizar questões estatísticas envolvidas nas previsões.

Tendo em vista o currículo estabelecido, não há suporte matemático para que os estudantes da Educação Básica possam compreender as tecnicalidades formais envolvidas em várias dessas previsões. Assim, uma alternativa para enfrentar a predominância do raciocínio determinístico na interpretação de resultados estatísticos é explorar reflexões de Inferência Estatística Informal.

O artigo está organizado da seguinte forma: na próxima seção, descrevemos as principais ideias envolvidas na Inferência Estatística Informal; em seguida, apresentamos uma atividade, em duas versões, para obter estimativas de $\pi$ que podem ser desenvolvidas na Educação Básica; concluímos o artigo com Considerações Finais e Referências.

\section{Inferência Estatística Informal}

Em 2008, o IASE- International Association for Statistical Education publicou um número especial de sua revista SERJ- Statistics Education Research Journal, dedicado à Inferência Estatística Informal (SERJ, 7-2, 2008). Esse número especial é uma boa leitura para quem desejar se aprofundar no tema, pois entre outras qualidades, apresenta trabalhos discutidos no fórum "Statistical Reasoning, Thinking and Literacy" (STRL-5) que reuniu pesquisadores da área de Educação Estatística em 2007 para refletir sobre Inferência Informal. 
DOI: http://dx.doi.org/10.33238/ReBECEM.2019.v.3.n.2.22509

Em trabalho publicado nesse número especial, acima mencionado, Zieffler et al., (2008) propõem uma estrutura conceitual para o entendimento e a pesquisa em Inferência Informal (chamada por eles de Raciocínio Inferencial Informal), além de sugerirem atividades. Também consultaram, nas áreas de Educação e Psicologia, trabalhos sobre Conhecimento Informal e Raciocínio Informal para concluirem:

[...] o desenvolvimento do conhecimento informal dos estudantes, relacionado à inferência estatística, pode facilitar sua transição para o entendimento das ideias formais de inferência (ZIEFFLER et al., 2008, p. 43, tradução nossa ${ }^{3}$ ). [...]

O raciocínio informal parece ser uma parte importante do Raciocínio Inferencial Informal, devido ao papel da evidência e da argumentação na elaboração de previsões e decisões estatísticas (ZIEFFLER et al., 2008, p. 44, tradução nossa ${ }^{4}$.

Os autores ainda sintetizam as várias definições de Raciocínio Inferencial Informal, utilizadas na literatura especializada, criando o que chamaram de definição prática (no contexto de aprendizagem):

[...] raciocínio Inferencial Informal é a forma como estudantes usam seus conhecimentos estatísticos informais para produzir argumentos que, baseados em amostras observadas, justificam inferências sobre populações desconhecidas (ZIEFFLER et al., 2008, p. 44, tradução nossa ${ }^{5}$ ).

Makar e Rubin (2009, p. 85), buscando ampliar a acessibilidade ao raciocínio inferencial com dados, consideram críticos os seguintes conceitos iniciais:

- Noção de incerteza e variabilidade, articulada através de linguagem, que quebre a convenção de afirmações com certeza matemática;

- Confiança no conceito de agregar (em oposição a pontos individuais) através do uso de generalizações sobre o grupo;

- Reconhecimento de mecanismo ou tendência que se estende além dos dados disponíveis;

• Evidência de raciocínio baseado no uso intencional de dados.

$\mathrm{Na}$ mesma direção de proporcionar meios para tornar mais acessíveis as idéias inferenciais, Wild et al., (2011) propõem atividades, com auxílio computacional e apelo visual, para realizar formas preliminares de inferência, isto é, inferências informais. Nessas atividades os recursos computacionais são usados de modo intenso, o que na Educação Básica brasileira muitas vezes é uma dificuldade. Como ilustração, apresentamos o resultado de uma dessas atividades na Figura 1, em que são realizadas

\footnotetext{
${ }^{3}$ No original: “[...] the nature of informal knowledge suggests that developing students' informal knowledge related to statistical inference may ease their transition to understanding formal ideas of inference".

${ }^{4}$ No original: "Informal reasoning seems to be an important part of Informal Inferential Reasoning (IIR) because of the role of evidence and argumentation in making statistical predictions and decisions".

${ }^{5}$ No original: "[...] the way in which students use their informal statistical knowledge to make arguments to support inferences about unknown populations based on observed samples".
} 


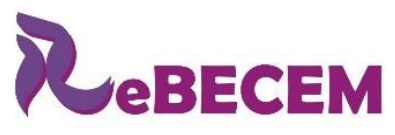

DOI: http://dx.doi.org/10.33238/ReBECEM.2019.v.3.n.2.22509
Revista Brasileira de Educação em

Ciências e Educação Matemática

várias repetições da coleta de uma amostra, de mesmo tamanho, retirada de uma população (no caso, altura de meninas de 13 anos). As repetições são feitas via simulação computacional e, para cada amostra obtida, é construído o respectivo gráfico box-plot. $\mathrm{O}$ tamanho da amostra varia de 10, 100 e 1000 observações. Ao criar uma imagem que mantém os gráficos anteriores, para cada tamanho de amostra, deixamos mais clara a noção de variabilidade dos resultados e sua dependência do tamanho da amostra.

Figura 1: Efeito visual de sucessivos gráficos box-plot

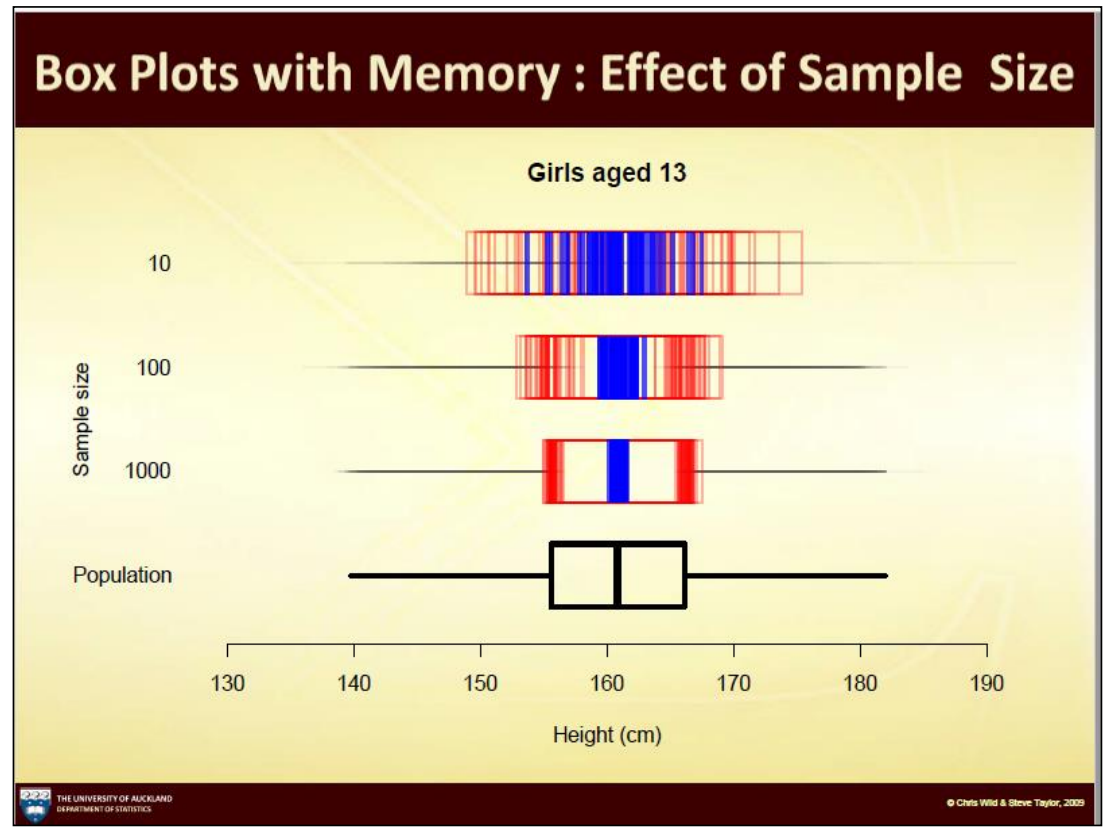

Fonte: https://www.stat.auckland.ac.nz/ wild/WPRH/

A Inferência Estatística Informal tem se consolidado como técnica importante. É uma forma de valorizar a análise descritiva de dados e uma ferramenta que auxilia o entendimento futuro da formalização inferencial. De modo geral, o desenvolvimento de projetos de coleta de dados pelos estudantes pode, também, proporcionar reflexões de Inferência Informal, com vantagens para o entendimento do uso de Estatística na sociedade. Convém ressaltar que é preciso sempre ter o cuidado de avaliar os limites das conclusões, verificando se é possível fazer afirmações informais sobre a população que originou os dados coletados.

No contexto brasileiro, trabalhos que mencionam explicitamente a Inferência Estatística Informal não são frequentes. Entretanto, notamos em diversas publicações a existência de artigos com relatos de atividades que incluem coleta de dados. Em alguns deles aparece o uso de Inferência Informal, ainda que de uma forma preliminar e não explícita, quando se pergunta sobre a extensão dos resultados obtidos para um universo maior do que a amostra, como em Cataneo, Martins e Burak (2016) e Guerra e Bisognin 


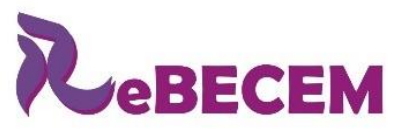

Revista Brasileira de Educação em

Ciências e Educação Matemática

DOI: http://dx.doi.org/10.33238/ReBECEM.2019.v.3.n.2.22509

(2016). Também, em feiras de divulgação científica e oficinas para complemento de formação de professores e de licenciandos em Matemática, a Inferência Informal está presente, conforme constatamos em roteiros dessas atividades. Por exemplo, em Cordani (2012), as atividades de estimação de tamanho de uma população de peixes e de avaliação da equiprobabilidade de uma moeda, incluem aspectos inferenciais sem o rigor da formalidade. Magalhães (2016), em texto relativo a um minicurso do XII ENEM, propõe que os estudantes comparem alturas dos gêneros, masculino e feminino, por meio da caixa central dos respectivos gráficos box-plot. A comparação é baseada em amostras que os estudantes coletam de um banco de dados simulando (ou imitando) retiradas ao acaso e com reposição. Não há formalidade inferencial nas conclusões, mas apenas avaliação visual dos resultados obtidos.

Cabe ainda mencionar duas dissertações, defendidas no programa de Mestrado Profissional em Ensino de Matemática do IME-USP, que buscaram explicitamente endereçar o uso de Inferência Estatística Informal no ensino regular. Em Camargo (2016), o autor propõe a comparação do desempenho de dois grupos. Inicialmente, apresenta uma solução usando Inferência Formal e, em seguida, sugere etapas para trabalhar o mesmo problema por meio de Inferência Informal. É enfatizado o auxílio de gráficos para tomar as decisões e os cuidados em justificar as conclusões. Também Rodrigues (2016), em sua dissertação, reflete sobre uma sequência didática para desenvolver conceitos de Estatística na $3^{\text {a }}$ série do Ensino Médio de uma escola particular. O autor, também professor da turma, iniciou a sequência com uma parte investigativa para motivar os estudantes. Houve uma coleta de dados e posterior análise, sendo que os conceitos estatísticos previstos no currículo foram sendo desenvolvidos ao longo da sequência didática. Na parte final da análise, os estudantes realizaram atividades de Inferência Informal. Os resultados revelam que houve reflexos positivos no aprendizado e na motivação dos estudantes.

\section{Atividade para avaliar $\pi$}

Conforme mencionado na Introdução, o primeiro método que se utiliza de Estatística para avaliar o valor de $\pi$ parece ter sido a Agulha de Buffon, cuja descrição mais detalhada pode ser obtida em https://pt.wikipedia.org/wiki/Agulha_de_Buffon. Nesse método, uma agulha é lançada ao acaso em uma mesa com linhas paralelas 
DOI: http://dx.doi.org/10.33238/ReBECEM.2019.v.3.n.2.22509

equidistantes e conta-se o número de vezes em que a agulha cruza alguma dessas linhas (ver Figura 2).

Figura 2: Várias realizações do experimento da Agulha de Buffon

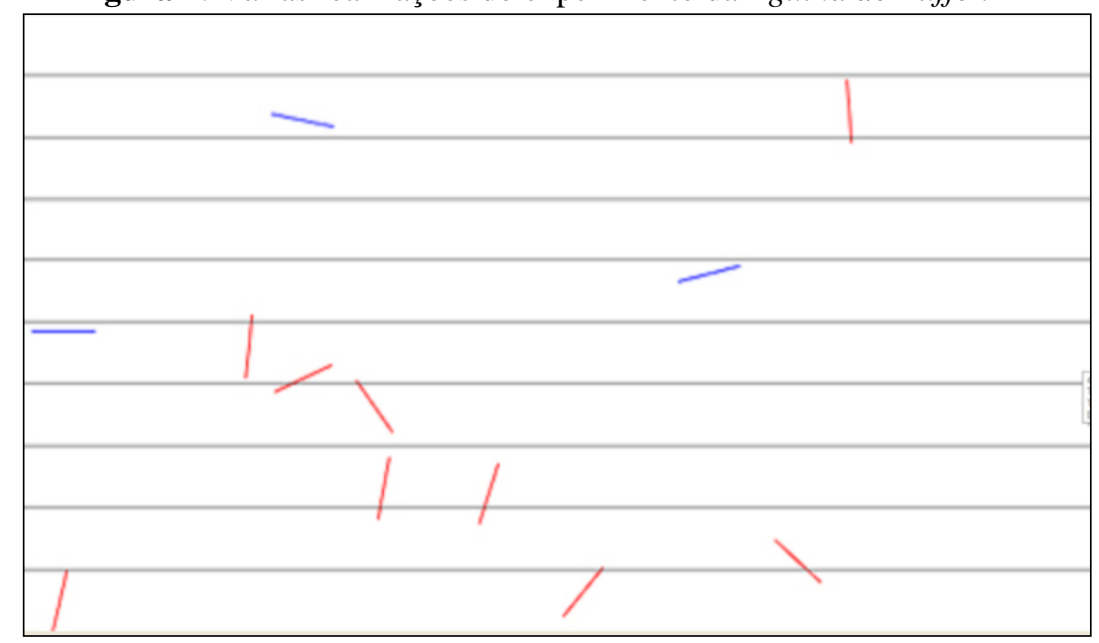

Fonte:https://www.metablake.com/pi.swf

Com base no tamanho da agulha e na distância entre as linhas, é possível estabelecer uma relação de $\pi$ com a probabilidade de cruzamento da agulha com as linhas. Calculando a frequência relativa dos cruzamentos da agulha com alguma das linhas, após vários lançamentos, obtemos uma estimativa dessa probabilidade que resulta em uma estimativa para $\pi$. A dedução da relação entre $\pi$ e a probabilidade, mencionada acima, envolve conceitos de Cálculo, com uso de coordenadas polares e integração, que são tópicos presentes apenas em currículos universitários da área de exatas. Apesar disso, mesmo na Educação Básica, não deixa de ser interessante mencionar que esse método existe e foi desenvolvido há muitos séculos. Nessa direção, um miniaplicativo que realiza os lançamentos da agulha e calcula as estimativas de $\pi$, pode ser encontrado em https://www.metablake.com/pi.swf.

A plena interação dos estudantes com as atividades que são realizadas é algo a perseguir no processo de ensino-aprendizagem e, dessa forma, buscamos desenvolver uma atividade para avaliar o valor de $\pi$ que usa conhecimentos curriculares da Educação Básica. Desa forma, são necessários os conceitos de área de círculo e de quadrado e a definição clássica de Probabilidade (número de casos favoráveis sobre número de casos possíveis), conteúdos esses, previstos para o $8^{\circ}$ e $6^{\circ}$ anos, respectivamente, de acordo com a BNCC (BRASIL, 2017). Também, será usada a expressão de distância entre dois pontos, conteúdo de Geometria Analítica, previsto no currículo do Ensino Médio. Cabe 
DOI: http://dx.doi.org/10.33238/ReBECEM.2019.v.3.n.2.22509

ressaltar que, pelas informações que dispomos, a atividade considerada foi originalmente elaborada pelo autor.

Antes de prosseguir com os outros detalhes da atividade, comentamos brevemente sobre o portal AtivEstat- Atividades de Estatística, coordenado pelo autor e acessado em https://www.ime.usp.br/ativestat. O portal AtivEstat tem acesso livre e é destinado a professores de Estatística de todos os níveis e, além de atividades, também contém indicações de outros portais de interesse. Cinco tipos de atividades estão presentes no portal e são descritos no Quadro 1.

Quadro 1: Tipo de atividades no AtivEstat

\begin{tabular}{|c|c|}
\hline Tipos de atividades & Descrição \\
\hline Sala de aula & $\begin{array}{c}\text { Atividades a serem desenvolvidas em classe e em algumas aulas, sem } \\
\text { necessidade de equipamento extra. }\end{array}$ \\
\hline Miniaplicativos & $\begin{array}{l}\text { Pequenos programas computacionais que realizam tarefas interativas com } \\
\text { o usuário. }\end{array}$ \\
\hline Planilhas & $\begin{array}{l}\text { Atividades para serem feitas em laboratórios de informática, ou em casa; } \\
\text { com auxílio de planilha computacional. }\end{array}$ \\
\hline Propostas de projeto & $\begin{array}{c}\text { Sugestões para desenvolvimento de projetos em grupos, com coleta de } \\
\text { dados e podem ser interdisciplinares. }\end{array}$ \\
\hline Filmes e vídeos & $\begin{array}{l}\text { Recursos multimídiausados em sala de aula ou em laboratório de } \\
\text { informática com acesso à internet. }\end{array}$ \\
\hline
\end{tabular}

Fonte: elaborado pelo autor

As atividades listadas no portal são produzidas por equipe própria ou vêm de outras fontes. Nesse caso, são mencionadas as respectivas referências para o devido crédito intelectual e, também, para a busca por mais detalhes teóricos ou práticos. Cada atividade tem uma breve descrição, informações sobre conceitos estatísticos envolvidos, nível de ensino adequado e tempo previsto de duração.

Um exemplo das informações disponíveis é apresentado na Figura 3, referente à atividade de sala de aula Quantos peixes têm no lago? 
Figura 3: Descrição da atividade SA07

\section{Alivis

\section{SA07: Quantos peixes têm no lago?}

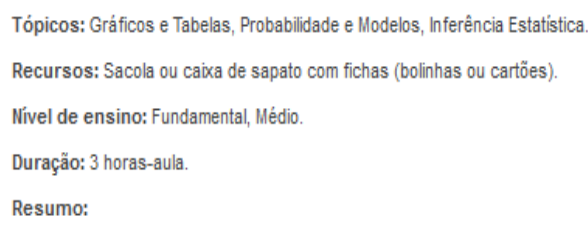

Nesta atividade, os estudantes simulam um procedimento para avaliar o número de peixes em um lago. Uma sacola simboliza o lago e as fichas os peixes. 0 número de fichas deve ser significantemente maior que o número de estudantes na sala. Discussões devem ser feitas sobre possíveis maneiras de estimar o número total de peixes. Uma delas será aplicada na atividade e é conhecida como o processo de "Captura e Recaptura", que será descrito a seguir. Um determinado número de peixes (fichas) é capturado, marcado e devolvido ao lago (sacola). Após os peixes (fichas) se misturarem bem, uma recaptura é feita. Assim, é possivel obter uma estimativa para o número de peixes no lago comparando duas proporções: a de peixes marcados em relação à população e a de peixes marcados escolhidos na recaptura. Após a estimação feita, os estudantes poderão, de fato, contar o número de fichas na sacola (peixes no lago). Atividade da oficina "Estatistica para todos ${ }^{n}$ desenvolvida pela professora Lisbeth K. Cordani.

Mais detalhes (pág. 15 a 22)

Fonte: Portal AtivEstat

Em seguida, apresentamos a descrição mais detalhada para cada uma das versões da atividade de avaliar o valor de $\pi$, presentes no portal AtivEstat. Apesar do referido portal já conter documentação sobre a atividade, salientamos que a ênfase aqui será destacar seu uso como uma prática de Inferência Estatística Informal.

Uma das versões é para aplicação em classe (versão Sala de aula), com relativamente pouco material de apoio, enquanto a outra versão requer o uso de recursos computacionais (versão Planilha).

\subsection{Atividade de sala de aula para avaliar $\pi$}

Em um quadrado de lado $a$, com um círculo inscrito (ver Figura 4), a área do quadrado é $a^{2}$, enquanto que a área do círculo é $\pi a^{2} / 4$. Logo, escolhendo um ponto ao acaso nessa figura, a probabilidade $p$, do ponto escolhido cair dentro do círculo, é o quociente das áreas acima (círculo por quadrado) e, assim temos $p=\pi / 4$.

A probabilidade calculada é denominada algumas vezes probabilidade geométrica. Ela corresponde a uma versão geométrica da definição clássica de probabilidade que é dada pelo quociente entre o número de casos favoráveis e o número de casos possíveis. Na probabilidade geométrica, o quociente se dá entre medidas de comprimento, área ou volume.

Apesar de $p$ ter sido calculada em $\pi / 4$, imaginamos, por um momento, que não conhecemos a constante $\pi$ e, portanto, não teríamos o conhecimento dessa probabilidade. 
DOI: http://dx.doi.org/10.33238/ReBECEM.2019.v.3.n.2.22509

De fato, por $\pi$ ser irracional, sempre trabalhamos com uma aproximação do seu valor com algumas casas decimais. A questão proposta aos estudantes é como obter um palpite para essa probabilidade, chamado de estimativa de $p$, e daí conseguir uma avaliação para $\pi$ multiplicando esse valor por 4.

Figura 4: Círculo inscrito em um quadrado

$\boldsymbol{a}$

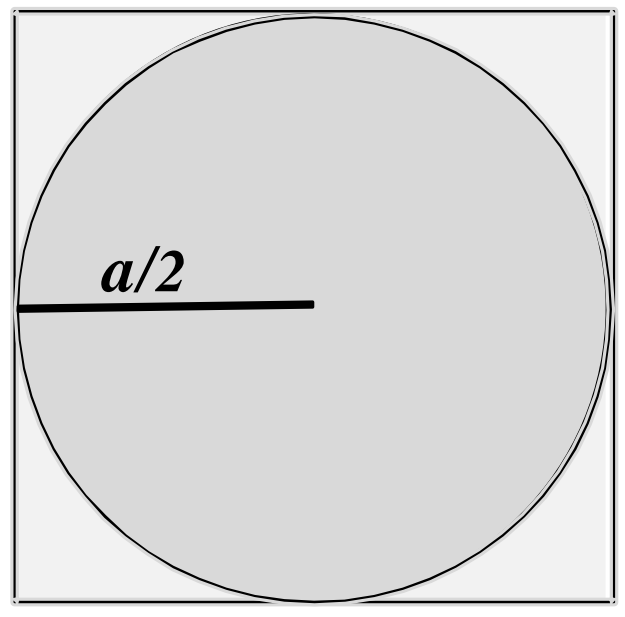

Fonte: elaborada pelo autor

Conforme já mencionado em referência ao texto de Makar e Rubin (2009), consideramos pré-requisito os estudantes conseguirem desenvolver os cálculos necessários de áreas e probabilidade. É claro que, dependendo da turma, isso pode requerer mais tempo de aula e diferentes níveis de intervenção do professor.

O desafio principal da atividade é refletir sobre formas de obter uma estimação da probabilidade de sortear, ao acaso, um ponto no círculo da Figura 4. Espera-se que os estudantes partam da sua intuição e, com auxílio do professor, possam chegar a uma proposta que envolva a necessidade de um procedimento amostral que leve em conta o agregado dos dados amostrados. Essas reflexões conduzidas pelo professor, bem como seus resultados, são ações de Inferência Estatística Informal, conforme mencionado em Zieffler et al., (2008). Note que, além dos procedimentos para a escolha de pontos ao acaso no quadrado indicado, a atividade envolve a discussão da noção de estimador.

Após a distribuição de cópias da Figura 4 (disponível no AtivEstat), dividimos a classe em grupos (2 ou 3 estudantes) e solicitamos que reflitam sobre como obter pontos, ao caso, nesse quadrado. É importante haver tempo para que os estudantes debatam suas ideias e, se necessário, devem receber auxílio do professor nas discussões.

Uma das formas de imitar um sorteio aleatório é soltar uma bolinha do alto em direção ao centro da figura e ver o local em que ela vai cair. Para facilitar o sorteio, dobre 
DOI: http://dx.doi.org/10.33238/ReBECEM.2019.v.3.n.2.22509

o papel para fazer bordas nos lados do quadrado, de modo que a bolinha caia dentro ou fora do círculo, mas sempre dentro do quadrado (ver Figura 5).

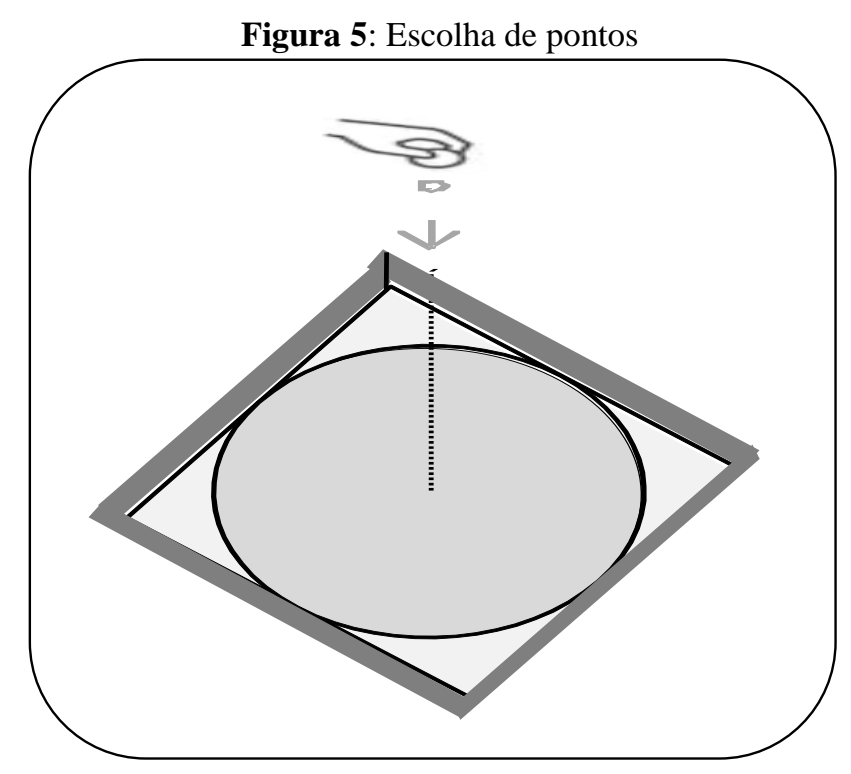

Fonte: elaborada pelo autor

Para fazer cada sorteio, a bolinha pode ser fornecida pelo professor ou mesmo "construída" pelos estudantes com um pedaço de guardanapo (por exemplo, um pedaço de $5 \times 5$, em centímetros, produz uma bolinha de tamanho adequado para a figura impressa em uma página).

Após o sorteio dos pontos, cabe a reflexão de como obter a estimativa da probabilidade desejada. A intuição dos estudantes deve ser trabalhada para que se conclua em usar a frequência relativa, ou proporção amostral, da ocorrência de pontos no círculo em relação ao total de pontos sorteados. O professor pode lembrar outras situaçõesfamiliares aos estudantes, em que se usa a frequência relativa para estimar uma probabilidade. Por exemplo, é comum estabelecer estimativas da probabilidade de atraso de uma pessoa para um encontro, com base nas vezes em que isso ocorreu anteriormente.

O número de pontos a serem sorteados deve ser discutido com a classe. Novamente, a intuição vai ser explorada para concluir que é preciso um número razoável de pontos para se ter melhores estimativas. Suponha que vamos obter 30 pontos, então o Quadro 2 pode ser preenchido.

Quadro 2: Resultados do sorteio

\begin{tabular}{|c|c|c|c|}
\hline Total de pontos & Node pontos no círculo & Freq.relativa $\left(f_{r}\right)$ & Estimativa de $\pi\left(4 f_{r}\right)$ \\
\hline 30 & & & \\
\hline
\end{tabular}

Fonte: elaborado pelo autor 


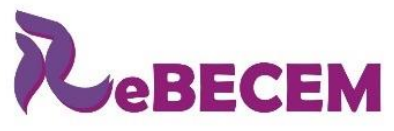

DOI: http://dx.doi.org/10.33238/ReBECEM.2019.v.3.n.2.22509

A partir do resultado de cada grupo, propomos uma discussão sobre a qualidade da avaliação de $\pi$. Nesse momento, esperamos comentários sobre variabilidade de resultados e aspectos referentes à amostragem (isto é, se os pontos foram mesmo aleatórios, se precisamos de mais pontos, etc.). Assim, são discutidos, de modo informal, diversos aspectos técnicos envolvidos na amostragem e na estimação. O mais importante da atividade não é a proximidade da estimativa ao valor $3,1415 \ldots$ (valor de $\pi$ ) mas a reflexão do estudante durante todo o desenvolvimento realizado na atividade.

Em acréscimo à discussão anterior, suponha que não levamos em conta as diferenças, entre os grupos, no procedimento de fazer o processo de amostragem. Dessa forma, podemos considerar que o sorteio tem o total de pontos de todos os grupos. Assim, a estimativa da probabilidade de sortear um ponto no círculo seria dada agora pelo quociente entre o total de pontos dos grupos dentro do círculo e o número total de pontos sorteados. Essa estimativa multiplicada por 4 produz uma nova avaliação do valor de $\pi$. Novamente, não temos garantia de obter estimativas que fiquem perto de $\pi$, mas a discussão envolvida nessa parte também vale a pena ser feita.

\subsection{Atividade de planilha para avaliar $\pi$}

A familiaridade com o uso de planilhas é necessária para desenvolvermos a atividade nessa versão e o professor precisa, caso necessário, preparar os estudantes previamente. Também, em adição ao conhecimento de cálculo de áreas e da definição clássica de probabilidade, necessitamos aqui das noções básicas de Geometria Analítica, em geral assunto do $1^{\circ}$ ano do Ensino Médio. Na Figura 6, o quadrado, com círculo inscrito, está representado em um plano cartesiano.

Como na versão sala de aula, vamos solicitar a reflexão dos estudantes para avaliar o valor de $\pi$. Iniciamos obtendo as áreas das figuras e a probabilidade de sortear ao acaso no quadrado, um ponto dentro do círculo. Em seguida, fazemos a discussão sobre a relação dessa probabilidade com o valor de $\pi$. Na versão planilha, a discussão referente à Inferência informal caminha de modo similar àquela já indicada na versão sala de aula. O desenvolvimento da atividade em pares (usando o mesmo computador) pode propiciar mais dinamismo na condução da atividade. 
DOI: http://dx.doi.org/10.33238/ReBECEM.2019.v.3.n.2.22509

Figura 6: Plano cartesiano

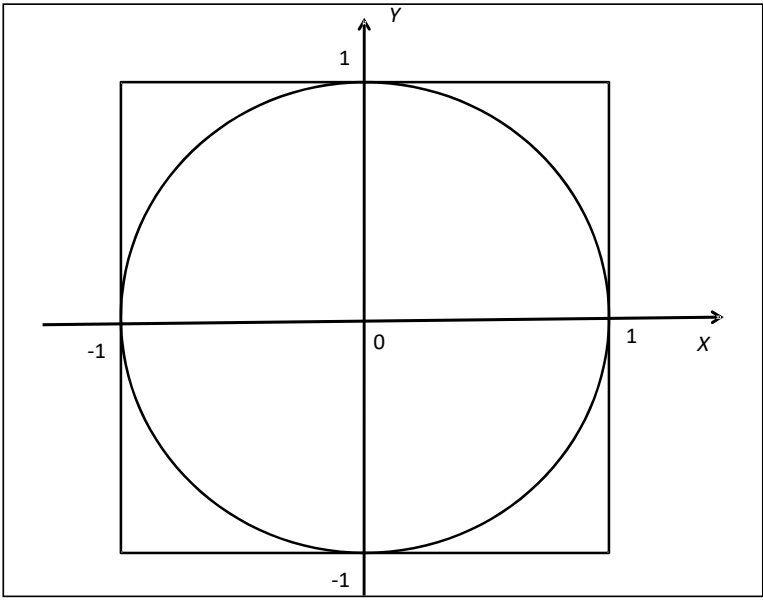

Fonte: elaborada pelo autor

Com o uso de planilhas, como Excel, Open Office ou Libre Office, a amostragem de pontos é facilitada. As planilhas podem gerar números aleatórios e realizar diversos cálculos, por meio de funções, cujas redações são similares nos vários programas computacionais. Em geral, anotamos os sucessivos cálculos em colunas e precisamos incluir o sinal de "=" antes do texto das funções.

Para escolher um ponto ao acaso no quadrado da Figura 6, obtemos suas coordenadas (abscissa e ordenada) por meio da geração de números aleatórios. Para verificar a posição do ponto no quadrado, calculamos sua distância à origem. Dessa forma, um ponto vai estar dentro do círculo se essa distância for menor ou igual a 1 . No Quadro 3, descrevemos as funções utilizadas (com a sintaxe do Excel).

Quadro 3: Funções para seleção de pontos no plano cartesiano

\begin{tabular}{|c|l|}
\hline \multicolumn{1}{|c|}{ Funções (Comandos) } & \multicolumn{1}{c|}{ Descrição } \\
\hline ALEATÓRIO( $)$ & Valor aleatório entre 0 e 1. \\
\hline Coluna A (abscissa):1-2*ALEATÓRIO() & Valor aleatório no intervalo [-1, 1] \\
\hline Coluna B (ordenada): 1-2*ALEATÓRIO() & Valor aleatório no intervalo [-1, 1]. \\
\hline $\begin{array}{l}\text { Coluna C (localização), na } 1^{\text {a }} \text { linha: } \\
\text { SE((RAIZ((A1^2)+(B1^2)) }<=1 ; 1 ; 0)\end{array}$ & $\begin{array}{l}\text { Valor } 1, \text { se o ponto (A1, B1) está no círculo; valor } \\
\text { 0, se está fora. }\end{array}$ \\
\hline
\end{tabular}

Fonte: elaborado pelo autor

Para efeito de estimação, é importante obter vários pontos, o que é relativamente simples quando usamos planilhas, basta clicar e deslizar o cursor. Assim, podemos obter $n$ linhas em que cada uma tem (nas Colunas A e B) as coordenadas de um ponto escolhido aleatoriamente dentro do quadrado. Se usarmos a função SOMA(), após a última linha da 


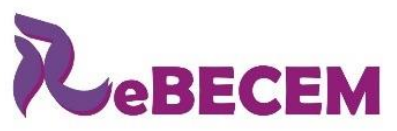

Revista Brasileira de Educação em

Ciências e Educação Matemática

DOI: http://dx.doi.org/10.33238/ReBECEM.2019.v.3.n.2.22509

Coluna $\mathrm{C}$, obtemos o número de pontos, dentre os $n$ sorteados,que estão dentro do círculo. Então, podemos calcular a frequência relativa da ocorrência de pontos no círculo, que é uma estimativa de $\pi / 4$. Portanto, multiplicando esse valor por quatro, obtém-se um valor aproximado de $\pi$.

Nessa versão da atividade, o número de pontos gerados pode, sem grandes esforços, ser aumentado. Dessa forma, surge a oportunidade de desenvolver outro aspecto da Inferência Informal referente à variabilidade das estimativas, conforme indicado em Wild et al., (2011).

Nesse sentido, podemos solicitar que os estudantes (ainda em pares) gerem e anotem os resultados de $\pi$, usando uma amostra de 10 pontos e, depois, sucessivamente, 50 e 100 pontos (talvez mais se desejarem). Recolhendo os diversos valores obtidos na classe, solicitamos que façam um box-plot desses valores para cada tamanho de amostra. Com mais pontos amostrados esperamos gráficos box-plot mais concentrados e, também, com valor de $\pi$ mais próximo do real. Entretanto, como o aleatório está sempre presente nos sorteios, não há garantias de melhor resultado, mas apenas uma maior probablidade de melhor resultado.

\section{Considerações finais}

O conteúdo previsto de Estatística na Educação Básica oferece a oportunidade do professor de Matemática fazer discussões referentes às previsões. Elas são uma forma de conexão com o cotidiano, que contribuem para desmistificar a Matemática como disciplina acessível apenas para alguns estudantes extraordinários.

A Inferência Estatística Informal é um caminho para realizar previsões quando não temos os instrumentos teóricos para uma abordagem formal da Inferência. É uma alternativa para os estudantes experimentarem o uso de Estatística e com isso se tornarem mais críticos das informações numéricas presentes na sociedade.

Cabe ao professor salientar aspectos de inferência que muitas vezes passam despercebidos e sem a devida ênfase. Solicitar conclusões, e comentários, que generalizem os dados coletados e avaliem os possíveis erros envolvidos, são momentos em que os estudantes estão refletindo num ambiente de previsão.

Em muitas das atividades para o ensino de Estatística é possível incluir as reflexões de Inferência informal. O portal AtivEstat contém diversas dessas atividades. Neste artigo, descrevemos uma delas, referente à avaliação de $\pi$. Ao buscar uma conexão 


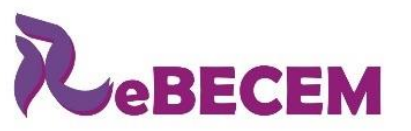

Revista Brasileira de Educação em

Ciências e Educação Matemática

DOI: http://dx.doi.org/10.33238/ReBECEM.2019.v.3.n.2.22509

com outros assuntos de Matemática, tentamos tomar vantagem da curiosidade envolvendo o número irracional $\pi$, para ampliar o entendimento de Estatística entre os estudantes.

De modo geral, o chamado Letramento estatístico da população depende de uma maior presença, e com mais qualidade, de tópicos de Estatística no currículo escolar. Atividades, como a discutida neste artigo, podem aumentar a motivação e a participação ativa do estudante na apropriação de conceitos estatísticos, contribuindo para o seu desenvolvimento pessoal nesses tópicos.

\section{Referências}

BOYER, C. B. História da Matemática. Tradução de Elza Gomide. 2. ed. São Paulo: Blucher, 1996.

BRASIL. Ministério da Educação. Secretaria de Educação Fundamental. Parâmetros Curriculares Nacionais Ensino Fundamental: ensino de primeira à quarta série. Brasília: MEC/SEF, 1997.

BRASIL. Ministério da Educação. Secretaria de Educação Fundamental. Parâmetros Curriculares Nacionais Ensino Fundamental: ensino de quinta à oitava série. Brasília: MEC/SEF, 1998.

BRASIL. Ministério da Educação. Secretaria de Educação Média e Tecnológica. Parâmetros Curriculares Nacionais. Brasília: MEC, 2000.

BRASIL. Ministério da Educação. Secretaria da Educação Média e Tecnológica. Parâmetros Curriculares Nacionais +: Ciências da Natureza e suas Tecnologias. Brasília: MEC, 2002.

BRASIL. Ministério da Educação. Governo Federal. Base nacional comum curricular para a Educação Infantil e o Ensino Fundamental. Brasília: MEC, 2017. Disponível em: http://basenacionalcomum.mec.gov.br/abase. Acesso em: 28 out. 2018.

CATANEO, G. S.; MARTINS, M. A.; BURAK, D. O ensino de Estatística mediado pela Modelagem Matemática. VIDYA, Santa Maria, v. 36, n. 2, p. 349-362.2016.

CAMARGO, A. R. A estatística na escola básica - uma prática de inferência informal. 2016. Dissertação (Mestrado Profissional em Ensino de Matemática) - Instituto de Matemática e Estatística, Universidade de São Paulo, São Paulo, 2016.

CORDANI, L. K. Estatística para todos- Atividades para a sala de aula. $1^{a}$ Edição. São Paulo: IME- USP, 2012.

GARFIELD, J. B.; BEN-ZVI, D. Developing students statistical reasoning: Connecting research and teaching practice. 1. ed. New York: Springer, 2008.

GUERRA, S. H. R.; BISOGNIN, V. Investigação Matemática na sala de aula: ensino de conceitos de Estatística para o $8^{\circ}$ ano do Ensino Fundamental. VIDYA, Santa Maria, v. 36, n. 2, p. 275-292. 2016. 
DOI: http://dx.doi.org/10.33238/ReBECEM.2019.v.3.n.2.22509

MAGALHÃES, M. N. Atividades para o ensino de Probabilidade e Estatística na Educação Básica. In: ENCONTRO NACIONAL DE EDUCAÇÃO MATEMÁTICA, 12., 2016, São Paulo. Anais... São Paulo: SBEM, 2016. p. 1-8. Disponível em: http://www.sbembrasil.org.br/enem2016/anais/pdf/5937_2749_ID.pdf. Acesso em: 22 maio. 2019.

MAKAR, K.; RUBIN, A. A framework for thinking about informal statistical inference. Statistics Education Research Journal, The Hague, v. 8, n. 1, p. 82-105, May. 2009.

RODRIGUES, V. A. Uma experiência de Inferência Estatística Informal na Escola Básica. 2016. Dissertação (Mestrado Profissional em Ensino de Matemática) - Instituto de Matemática e Estatística, Universidade de São Paulo, São Paulo, 2016.

ZIEFFLER, A. et al. A framework to support research on informal inferential reasoning. Statistics Education Research Journal, The Hague, v. 7, n. 2, p. 40-58, November .2008.

WILD, C. J. et al. Towards more accessible conceptions of statistical inference. J. R. Statist. Soc. A, London, v. 174, n. 2, p. 247-295, apr. 2011.

Recebido em: 29 de maio de 2019.

Aceito em: 16 de agosto de 2019. 